\title{
RNA sequencing analysis reveals the competing endogenous RNAs interplay in resected liver cancer patients who received interferon-alpha therapy
}

\section{Yibin Wu}

Fudan University Shanghai Cancer Center

\section{Longrong Wang}

Fudan University Shanghai Cancer Center

\section{Xiaoshuang Wang}

Fudan University Shanghai Cancer Center

Yiming Zhao

Fudan University Shanghai Cancer Center

\section{Anrong Mao}

Fudan University Shanghai Cancer Center

\section{Ning Zhang}

Fudan University Shanghai Cancer Center

Jiamin Zhou

Fudan University Shanghai Cancer Center

\section{Qi Pan}

Fudan University Shanghai Cancer Center

\section{Weiping Zhu}

Fudan University Shanghai Cancer Center

Lu Wang ( $\sim 20111230020 @ f u d a n . e d u . c n$ )

Fudan University Shanghai Cancer Center https://orcid.org/0000-0001-8656-2183

\section{Primary research}

Keywords: Hepatocellular carcinoma, Interferon-alpha, RNA sequencing, Competing endogenous RNAs, MARCH3, Tumor-infiltrating

Posted Date: March 18th, 2021

DOl: https://doi.org/10.21203/rs.3.rs-254813/v1

License: (c) (1) This work is licensed under a Creative Commons Attribution 4.0 International License. Read Full License 


\section{Abstract \\ Background}

Interferon-alpha (IFN- $a$ ) is a general therapeutic regimen to be utilized in HCC. However, regulatory mechanisms of IFN-a on competing endogenous RNAs (ceRNAs) level in HCC are rarely understood.

\section{Methods}

HCC patients with and without IFN-a treatment were calculated to analyze the expression profile of mRNA, IncRNA, miRNA, and circRNA by RNA sequence, and significant differential expression (DE) of these types of RNAs were screened out for the following analysis. A ceRNA regulatory network was constructed to explore effects of IFN-a intervention on HCC. Furthermore, the potential prognostic factors among these DE RNAs were identified.

\section{Results}

In total, 60 (0.37\%) mRNAs, $23(0.08 \%)$ circRNAs, 15 (0.11\%) IncRNAs, and $23(0.91 \%)$ miRNAs were differentially expressed in patients who received IFN-a treatment. A ceRNA regulatory network including a circRNA-miRNA-mRNA network which composed of 4 up- and 10 down-regulated circRNAs, 8 up- and 5 down-regulated miRNAs, 28 up- and 9 down-regulated mRNAs, and a IncRNA-miRNA-mRNA network which composed of 10 up- and 3 down-regulated IncRNAs, 11 up- and 5 down-regulated miRNAs, 28 upand 10 down-regulated mRNAs was constructed. Gene enrichment and pathway analysis revealed that the ceRNA network was associated with immune-related pathway and corresponding molecular function in patients who accepted IFN- $a$ treatment. Next, we identified 3 most relevant to IFN-a treatment to HCC among these DE RNAs, namely FAM20A,IGFBP4 and MARCH3, as the prognostic factors of HCC. Furthermore, MARCH3 expression was positively correlated with infiltrating levels of CD4 + T and CD $8+T$ cells, macrophages, neutrophils, and dendritic cells (DCs) in HCC. MARCH3 expression showed strong correlations with diverse immune marker sets in HCC.

\section{Conclusion}

Our data discovered a novel ceRNA network in HCC patients receiving IFN-a therapy, which might lay the foundation for better understand the regulatory mechanism of IFN-a treatment.

\section{Background}

Hepatocellular carcinoma (HCC), one of the most serious malignancies, is highly prevalent in China [1]. Although surgery is one of the curative-intent options to patients with early-stage disease, the high recurrence rate (50\% at 3 years and $70 \%$ at 5 years) greatly reduces the effectiveness of this method [2- 
4]. Thus, adjuvant therapy is increasing attractive and becomes the research focus. Interferon-alpha (IFNa) is an antiviral cytokine which is with known anti-proliferative and immunomodulatory properties against malignant tumors [5], and has been published that could inhibit tumor metastasis in nude mice bearing human HCC xenografts [6], and in our clinical trials [7]. However, the characteristics of patients who can benefit from IFN-a are not consistent.

Yang and his team have reported that higher IFIT3 expression in tumor tissue than normal tissue can be regarded as a factor to predict response of IFN-a therapy [8]. Retinoic acid-inducible gene-I (RIG-I), another interferon-stimulated gene, has also proven as a predictive factor. Namely, patients with low RIG-I expression always presented poorer response and shorter survival to IFN-a therapy[9]. Meanwhile, we found that dihydropyrimidine dehydrogenase (DPYD), a pyrimidine catabolic enzyme, was dosedependently downregulated by IFN- $a$ in HCC tissues, and might be a potential prognostic biomarker and a therapeutic target for HCC [10]. Although many research have screened the potential patients benefited from IFN-a therapy, no agreement is reached.

Competing endogenous RNA (ceRNA) network is an entrant which can indirectly regulate mRNAs via competitively binding miRNAs. And in this theory, ceRNA refers to all the transcripts which can be used as the targets of miRNAs, namely including circular RNA (circRNA), pseudogene RNA, and long non-coding RNA (IncRNA)[11]. The mechanisms on regulating IFN-a mRNA level by ceRNA network have been widely reported. Specifically, Tominori and his colleagues have published that according to binding with miR1270, the ceRNA including IFN- $\alpha 1$ antisense RNA (AS) and cellular mRNAs precisely maintains the level of the type-I IFN and functions on the innate immune system[12]. In vitro experiments also have indicated that several IncRNA were differentially expressed to induce the IFN pathway under the IFN-stimulation [13]. In addition, ceRNA has also been researched in the area of liver cancer. Based on The Cancer Genome Atlas (TCGA) database, Zhao et al has discovered that a HCC-related ceRNA network which can be used as the prognostic factor to reveal cancer progression [14]. Another larger-clinical-sample research further indicated that the ceRNA network might aid to elucidate the mechanism of HCC pathogenesis [15]. Although as previously mentioned, less results have been announced to probe the mechanism on ceRNA regulating the IFN-associated pathway in HCC based on clinical samples.

Currently, utilizing the clinical samples, we try to probe the possible mechanism on how the IFN-a functions on cancer cells based on RNA sequencing. TIMER and GEPIA was used to further analyze the correction between MARCH3 expression and tumor-infiltrating in $\mathrm{HCC}$. The results may lay the foundation on better comprehending the functional process of IFN-a treatment on HCC, and screening potentially beneficial patients who received the IFN-a therapy.

\section{Materials And Methods}

\section{Patients}

Totally, 4 patients received surgical treatment at Fudan University Shanghai Cancer Center (Shanghai, China) were enrolled in this study. Two weeks after surgery, four samples from these patients were 
collected before interferon-alpha treatment (group A). While the other four samples were immediately collected from these resected patients injected with interferon-alpha (30 mg twice a week; recombinant human interferon a-2b; Shenzhen Kexing Bioengineering Co., Ltd.) after two weeks (group B). The clinicpathological characteristics of these patients are shown in Supplementary Table 1. Prior patient consent were obtained, and the study was approved by the Ethics Committee of Fudan University Shanghai Cancer Center.

\section{Isolation of peripheral blood mononuclear cell (PBMC) and sequencing}

PBMCs were respectively isolated from the peripheral blood of all the participants based on the previous method with some modifications [16]. Firstly, the peripheral blood was layered and centrifuged $(950 \mathrm{~g}$, $30 \mathrm{~min})$. Then the Ficoll-Histopaque layer was collected and stored for the following experiments. The trypan blue exclusion test was utilized to assay the cell viability.

Total RNA was isolated from the extracted PBMC by Trizol reagent (Invitrogen, USA) following the manufacturer's protocol. With the aid of 2100 Bioanalyzer (Agilent, USA) and NanoDrop Spectrophotometer (NanoDrop, USA), the RNA quality was detected.

\section{RNA library construction and sequencing}

The TrueSeq small RNA library prep kit (Illumina, USA) was utilized to construct the RNA (RNA) library followed the manufacturer's protocol. Briefly, after removing the ribosomal RNA, mRNA, miRNA and circRNAs were respectively fragmented and synthesized cDNAs. After purifying, connecting with adaptors, they was used as the templates, and the single-end sequencing was worked on the HiSeq4000 sequencer (IIlumina, USA).

\section{Date processing and differentially expressed RNAs screening}

Raw reads were screened with an in-house pipeline which had been internally validated. To acquire the clean reads, adaptor, low quality, and high unknown bases were trimmed. Genome GRCh38 was utilized as the human reference background and the analyzing tool was Bowtie[17] or Tophet[18]. The expression levels of RNAs were counted by using the reads per kilobase transcriptome per million mapped reads (RPKM) method. The differentially expressed (DE) RNAs (miRNAs, mRNAs, or circRNAs) between samples were identified by the DEGseq or Cufflinks package under the R environment. The cut-off cirteria was designed as log2 (Fold_change) $>2$ with the p value $<0.01$. After that, Cytoscape software (version 3.7.0) was utilized to construct the ceRNA network. 


\section{Bioinformatics analysis}

Firstly, the miRWalk (http://mirwalk.umm.uni-heidelberg.de/) and TargetScan (http://www.targetscan.org/mamm_31/) software were respectively utilized to predict the target genes and target circRNAs for the DEmiRNAs. The starbased website (http://starbase.sysu.edu.cn/) was utilized to predict the target genes for DEcircRNAs. Then Gene Ontology (GO) enrichment and Kyoto Encyclopedia of Genes and Genomes (KEGG) pathway were analyzed on these DERNAs, including DEmRNAs and the target genes of DEmiRNAs and DEcircRNAs. Merely when $P$ value $<0.05$, the analysis results were regarded as owning the statistical significance.

Secondly, the possible functional relationships between DERNAs were further probed. Significantly DEmRNAs and DEcircRNAs were analyzed the Pearson correlation coefficient to determine whether presented co-expressed, and the Pearson correlation coefficient index was designed over than 0.9 with an adjusted $p$ value $<0.1$. The whole ceRNA network including the miRNA-circRNA regulatory network, the miRNA-target gene regulatory network, and the circRNA-mRNA co-expression network were constructed.

\section{TIMER database analysis}

TIMER is a comprehensive resource for systematic analysis of immune infiltrates across diverse cancer types (https://cistrome.shinyapps.io/timer/). It includes 10,897 samples across 32 cancer types from The Cancer Genome Atlas (TCGA) to estimate the abundance of immune infiltrates. We used TIMER to investigate the correlations between $\mathrm{MARCH} 3$ expression with the abundance of immune infiltrates, including B cells, CD4+ T cells, CD8+ T cells, neutrophils, macrophages, and dendritic cells, via gene modules. In addition, we also analyzed the correlations between MARCH3 expression and gene markers of the different tumor infiltrating immune cells. The relative gene markers are ever reported in literature.

\section{Gene correlation analysis in GEPIA}

GEPIA (http://gepia.cancer-pku.cn/) is an online tool for analyzing the RNA sequencing expression data of 9,736 tumors and 8,587 normal samples from the Cancer Genome Atlas and the Genotype Tissue Expression (GTEx) projects. We further confirmed the correlation of MARCH3 with T cell exhaustion via analyzing GEPIA dataset. The Spearman method was used to determine the correlation coefficient.

\section{Statistical analysis}

Baseline characteristics of the two groups were compared using the Chi square test or Fisher's exact test. Survival curves were depicted by the "Survival" package under the R environment with the Kaplan-Meier method. The survival data originated from the cBio Cancer Genomics Portal 
(https://www.cbioportal.org/). The p value $<0.05$ was regarded as the cut-off threshold for significant meaning. All statistical analyses were performed using SPSS 20.0 software (IBM, USA).

\section{Results}

\section{Patient's characteristics}

The clinical characteristics of the enrolled population were listed in Supplementary Table 1. All the 4 patients were diagnosed as the hepatocellular carcinoma (HCC). The median age for all patients was 52 years. All of the patients had HBV viral infection, and the sex was all male. All the patients had a normal $\mathrm{BMI}$ and good ECOG performance status. No patients had received neoadjuvant therapy, and all the patients presented negative surgical margin.

\section{Differentially expressed mRNA, miRNA and circRNA}

Totally, 15806 mRNAs, 2464 miRNAs, 12395 IncRNAs and 15769 circRNAs were discovered in group A, and the corresponding number were $15875,2318,12710$ and 18911 in group $B$, respectively. Comparing with group A, 60 (0.37\%) mRNAs, 23 (0.08\%) circRNAs, 15 (0.11\%) IncRNAs, and 23 (0.91\%) miRNAs were differentially expressed in group B. Finally, we identified top 8 of mRNAs, circRNAs and IncRNAs that associate HCC patients who IFN-a therapy as showed in heat map (Fig. 1a, b, c).

\section{The ceRNA network}

The ceRNA regulatory network was described as follows. In the up-regulated circRNA-miRNA-mRNA network, there were 4 circRNAs, 28 mRNAs and 5 miRNAs, and in the corresponding down-regulated network, 10 circRNAs, 9 mRNAs, and 8 miRNAs were involved (Fig. 2a). The up-regulated IncRNA-miRNAmRNA network included 10 IncRNAs, 28 mRNAs and 5 miRNAs, and the down-regulated network contained 3 IncRNAs, 10 mRNAs and 11miRNAs (Fig. 2b).

\section{Functional annotation of the ceRNA network}

The GO and KEGG analysis were performed to understand the biological function in the DE-ceRNA network. The result of GO analysis was shown in Figure 3. Firstly, for circRNA-miRNA-mRNA network, the genes were mainly enriched in synapse, external side of the plasma membrane, and juxtaparanode region of axon; the most enriched GO terms in molecular function (MF) were chemokine receptor activity, lipoprotein transporter activity and P2Y1 nucleotide receptor binding; in additional, humoral immune response, vocalization behavior, chemokine-mediated signaling pathway and immune response were the 
mainly enriched biological process (BP). For IncRNA-miRNA-mRNA network, the enriched terms of cellular component (CC), MF and BP were basically similar with that of the circRNA-miRNA-mRNA network.

Then, we performed the pathway enrichment analysis of the ceRNA network (Fig. 4a, b). The up-regulated RNAs networks including the circRNA-miRNA-mRNA and IncRNA-miRNA-mRNA were both enriched in the five pathways, namely hematopoietic cell lineage, cell adhesion molecules (CAMs), chemokine signaling pathway, cytokine-cytokine receptor interaction, and influenza A. And the down-regulated RNAs network mainly mapped on the following pathways, including nicotinate and nicotinamide metabolism, asthma, and allograft rejection.

\section{Survival analysis of pivotal genes}

We utilized the data from TCGA to perform the survival analysis of pivotal genes (Fig. 5a, b, c). Only three genes among the DE-ceRNA regulatory network were related with the prognosis. Specifically, the results indicated that patients with FAM20A high expression presented a significantly longer survival time than the rest $(P=0.0012)$, and the same results were also observed in patients with IGFBP4 $(P=0.0083)$ and MARCH3 ( $P=0.049)$.

\section{MARCH3 expression is correlated with immune infiltration level in HCC}

To further analyze the effect of MARCH3 gene on HCC occurrence, we used TIMER to examine the expression lever of MARCH3 in liver cancer. The data showed that MARCH3 mRNA was highly expressed in normal tissues than tumor tissues (Fig. 6a). Tumor-infiltrating lymphocytes are an independent predictor of sentinel lymph node status and survival in cancers. Tumor purity is an important factor that influences the analysis of immune infiltration in clinical tumor samples by genomic approaches, and TIMER and GEPIA have most of the homologous data from TCGA. By analyzing data from TIMER, we found that MARCH3 had a negative correlation with tumor purity in LIHC. And MARCH3 expression level also correlated with poorer prognosis and high infiltration in $\mathrm{LIHC}$, as the results showed that MARCH3 had positive related with $B$ cell $(r=0.365, p=2.64 e-12), C D 8+T$ cell $(r=0.312, p=3.53 e-9), C D 4+T$ cell $(r=0.417, p=3.23 e-21)$, macrophage $(r=0.514, p=2.09 e-24)$, neutrophil $(r=0.437, p=1.72 e-72)$ and dendritic cell $(r=0.44, p=9.85 e-16)$ (Fig. 6b). Next, we explored the effects of the copy number variation (CNV) of MARCH3 on the immune infiltration, the results showed that high amplication of MARCH3 could increase

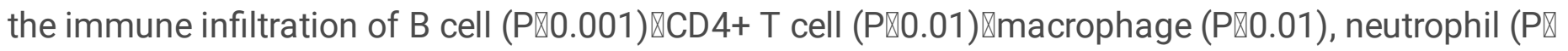
$0.0001)$ and dendritic cell (Pख0.01), while arm-lever gain of $M A R C H 3$ could increase the immune infiltration of $C D 4+T$ cell $(P \llbracket 0.001) \varangle$ macrophage ( $P \llbracket 0.01)$, neutrophil (Pख0.01) (Fig. 6c). This finding strongly indicates MARCH3 plays a crucial role in immune infiltration in HCC. 


\section{Correlation analysis between MARCH3 expression and Immune marker sets}

In order to analyze the correlation between MARCH3 and the diverse immune infiltrating cells, we further investigate the relationships between MARCH3 and immune marker sets of various immune cells of LIHC in the GEPIA and TIMER databases. We studied the associations between MARCH3 expression and immune marker genes of different immune cells, such as CD8+ T cells, $T$ cells (general), B cells, monocytes, TAMs, M1 and M2 macrophages, neutrophils, NK cells and DCs in LIHC, and the different functional T cells included Th1 cells, Th2 cells, Tfh cells, Th17 cells, Tregs and exhausted T cells. After the correlation adjustment by purity, the results showed that MARCH3 expression was significantly correlated with most immune marker sets of various immune cells and different T cells in HCC (Table 2).

Interestingly, we also found that MARCH3 expression had strong positive correlations with the expression levels of most marker sets of T cell exhaustion (PD-1 and CTAL4) in HCC (Fig. 6d, P囚0.0001). We also examined the correlation by analyzing a HCC TCGA data from GEPIA database, and our data showed that $M A R C H 3$ expression was positively associated with the marker sets of PD-1 ( $R=0.43 ; P \llbracket 0.0001)$ and CTAL $(R=0.42 ; P \llbracket 0.0001)$ (Table 3), whose results were consistent with TIMER analysis. These findings suggest that MARCH3 may regulate the immune function to affect the HCC occurrence.

\section{Discussion}

In this study, to clarify the regulatory mechanism of IFN-a therapy on HCC, we analyzed the RNA expression profile by RNA sequencing. According to comparing RNA profile of patients with IFN-a or not, totally 60 (0.37\%) mRNAs, $23(0.08 \%)$ circRNAs and 23 (0.91\%) miRNAs were differentially expressed. Then a ceRNA network included the circRNA-miRNA-mRNA and IncRNA-miRNA-mRNA were constructed. Besides, the biological and functional attributes of these significantly differentially expressed RNAs were analyzed by GO and KEGG databases. Immune-related biological progresses were significantly more enriched. Survival analysis showed that three potential prognostic associated genes, namely FAM20A, IGFBP4 and MARCH3. Finally, we further analyzed the correlation between MARCH3 and immune infiltrate in HCC. To our knowledge, this is the first publication to probe the ceRNA network of IFN-a therapy in HCC and identify the effect of MARCH3 associating with immune function on LIHC.

Of the genes which were involved in the ceRNA network, $\mathrm{GO}$ enrichment analysis indicated that these RNAs were mainly enriched in the synapse, external side of the plasma membrane, and juxtaparanode region of axon. IFN-a, a proinflammatory cytokine, which is commonly produced by natural killer cells and T-lymphocytes [19]. As under the normal conditions, these cells minimally entry into the central nervous system, IFN- $a$ is generally not detected in the brain [20]. But several research have revealed that the high expression level of IFN-a can cause hippocampal development and abnormal cerebellar [21, 22]. The effects of IFN- $a$ on neurons is increasingly drawn attention. McHugh has published a comment that IFN-a can stimulate synapse loss[23]. And based on a mouse model, IFN-a inhibits the dendritic outgrowth which may lead to decreasing the rate of synapse formation [24]. Besides, IFN-a has also been proven to provide the enhanced axon protection [25]. And for localizing in the plasma membrane, this result has 
been widely accepted as many plasma membrane proteins, like tetherin and IFITM3, are identified in the interferon-treated cells [26]. In addition, the biological process of GO term and pathway have mapped the immune-related signaling. As we all know, the IFN-a is a family of cytokine mediators which involves in mediating the cellular immune system [27]. The normal interferon pathways always start with binding ligand to form a ternary complex, and then followed by activating the downstream signaling, like mediating the Janus tyrosine kinases [28]. Then the Toll-like receptors, tissue-destructive cytokines, inflammatory factors, cytokines are regulated to activate the STAT. Subsequently, the function of immune effector genes, like chemokines, phagocytic receptors, antiviral proteins, antigen-presenting molecules, are activated [29]. In current research, we discovered that the significantly differential expression of ceRNA mainly mapped on the immune-related pathway, and this result further proven the importance of the relationship between IFN- $a$ and immune pathway.

FAM20A, encoding one of the "family with sequence similarity 20" (FAM20) proteins, function in the secretory signaling to promote protein phosphorylation. In human mesenchymal stem cells, previous paper had proven that this protein presented high expressed level following the IFN-a treatment[30]. Although this protein is originally identified from the hematopoietic cell, FAM20A presents a quite restricted high expression in liver tissue [31]. In addition, based on the data from the Human Protein Altas (HPA, https://www.proteinatlas.org/ENSG00000108950-FAM20A/pathology/liver+cancer\#ihc), FAM20A displayed low expressed level in LIHC. And what' more, FAM20A is a prognostic factor in LIHC. However, another largely clinical cohorts are needed to further test the above results. IGFBP4 is a specific insulinlike growths factor (IGF) binding protein which binds to IGF or not to tune the cellular activities, like cell proliferation and migration [32,33]. In vitro experiment has revealed that the IFN-a can increase the mRNA and protein level of IGFBP4 [34]. And this protein, functioning as a tumor suppressor, participates in driving epigenetic reprogramming of the hepatic carcinogenesis [35]. Although currently there is no research on the relationship between IGFBP4 and prognosis in liver cancer, it has been reported to be associated with prognosis in various cancer types, like lung cancer [36]. Our work first revealed that patients with higher IGFBP4 expression had a longer survival time than that with lower IGFBP4 expression. Membrane-associated RING-CH-3 (MARCH3) is one of the membrane-associated MARCH family members which functions as a negative regulator of adaptive immunity [37]. The whole MARCH family includes 11 members, and 9 of them are transmembrane proteins. Many of these family members have been revealed to associate with prognosis. By researching on a mouse model, targeting MARCH1 exhibited significant inhibition of the growth of HCC [38], and MARCH8 has also played a crucial role in NSCLC against carcinogenesis and progression by validating in clinical samples [39]. In this work, we first found that MARCH3 was highly expressed in $\mathrm{HCC}$ and patients with higher MARCH3 expression suffered shorter survive, which suggested MARCH3 as an unfavorable prognostic factor in HCC. Next, we found that $\mathrm{MARCH} 3$ expression was related with diverse immune infiltration levels in HCC. Our results demonstrated that there was a strong positive relationships between MARCH3 expression level and CD4 + T cells, macrophages, neutrophil and DCs, and significantly positive correlations between B cells, CD8 + T cells and MARCH3 expression in HCC. Moreover, the correlations between MARCH3 expression and the marker genes of immune cells implicated the role of MARCH3 in regulating tumor immunology in HCC. 
We found that MARCH3 expression was highly correlated with the marker sets of T cell exhaustion, namely PD-1 and CTAL4. These correlations could be indicative of a potential mechanism where $M A R C H 3$ regulates $T$ cell functions in $\mathrm{HCC}$. Together these findings suggest that the MARCH3 plays an important role in recruitment and regulation of immune infiltrating cells in HCC.

Although the current research constructed a ceRNA network in $\mathrm{HCC}$ for probing the regulation mechanism of IFN-a for the first time, potential limitations existed. First, merely 8 HCC blood samples were obtained from the clinical patients which might lead to the selection bias. Second, in the current research, we haven't provided the validation data on the discovered results based on the downstream experiments. Anyway, a clinical cohort in larger patient sizes and a following validated experiment are unmet needed in the future.

\section{Conclusions}

We constructed a ceRNA network and analyzed the biological function of RNAs with significantly differential expression in HCC on researching the effect from IFN-a. The results indicated that immunerelated pathways played crucial role in participating in IFN-a treatment. Three genes (FAM20A, IGFBP4 and MARCH3) were identified as the prognostic markers, respectively. Finally, we identified MARCH3 as a vital factor in recruitment and regulation of immune infiltrating cells in HCC. These results laid the foundation on understanding the regulatory mechanism of IFN-a treatment.

\section{Abbreviations}

HCC: hepatocellular carcinoma; IFN-a: Interferon-alpha; ceRNAs: competing endogenous RNAs; DE: differential expression; RIG-I: Retinoic acid-inducible gene-l; DPYD: dihydropyrimidine dehydrogenase.

\section{Declarations}

\section{Acknowledgements}

Not application

\section{Author contributions}

Yibin Wu, Longrong Wang. and Xiaoshuang Wang performed the experiments and drafted the manuscript. Yiming Zhao, Anrong Mao and Jiamin Zhou analyzed the data and edited the paper. Qi Pan and Ning Zhang collected the clinical samples. Weiping Zhu and Lu Wang were responsible for designing the experiments and supervising the study. All authors read and approved the final version of the manuscript. 


\section{Funding}

This work was jointly supported by National Natural Science Foundation of China (81874056, 81874182, and 81372314), the Public Health Bureau Foundation of Shanghai $(201840019,201940043)$, the National Key Project of China (2017ZX10203204-007-004), Shanghai Shenkang clinical science and technology innovation project (SHDC12019X19), and the Research Talent Foundation of Fudan University Shanghai Cancer Center (YJRC1604, YJQN201932).

\section{Availability of data and materials}

The data sets generated and analyzed during the current study are available from the corresponding author on reasonable request.

\section{Declaration}

\section{Ethics approval and consent to participate}

The study was approved by the Ethics Committee of Fudan University Shanghai Cancer Center. Written informed consent was obtained from each participant.

\section{Consent for publication}

All persons designated as authors for authorship and have approved the final version of the manuscript.

\section{Competing interests}

The authors declare no conflict of interest.

\section{Author details}

${ }^{1}$ Department of Hepatic Surgery, Fudan University Shanghai Cancer Center, Shanghai Medical College, Fudan University, Shanghai 200032, PR China

\section{References}


1. Bray F, Ferlay J, Soerjomataram I, Siegel RL, Torre LA, Jemal A: Global cancer statistics 2018: GLOBOCAN estimates of incidence and mortality worldwide for 36 cancers in 185 countries. $C A$ : a cancer journal for clinicians 2018, 68(6):394-424.

2. Bruix J, Sherman M: Management of hepatocellular carcinoma: an update. Hepatology 2011, 53(3):1020-1022.

3. EASL-EORTC clinical practice guidelines: management of hepatocellular carcinoma. J Hepato/ 2012, 56(4):908-943.

4. Kawaguchi Y, Honda G, Endo I, Cherqui D, Kokudo N: Current Technical Issues for Surgery of Primary Liver Cancer. Liver cancer 2016, 6(1):51-58.

5. Belardelli F, Ferrantini M, Proietti E, Kirkwood JM: Interferon-alpha in tumor immunity and immunotherapy. Cytokine \& growth factor reviews 2002, 13(2):119-134.

6. Wang L, Tang ZY, Qin LX, Wu XF, Sun HC, Xue Q, Ye SL: High-dose and long-term therapy with interferon-alfa inhibits tumor growth and recurrence in nude mice bearing human hepatocellular carcinoma xenografts with high metastatic potential. Hepatology 2000, 32(1):43-48.

7. Sun HC, Tang ZY, Wang L, Qin LX, Ma ZC, Ye QH, Zhang BH, Qian YB, Wu ZQ, Fan J et al: Postoperative interferon alpha treatment postponed recurrence and improved overall survival in patients after curative resection of HBV-related hepatocellular carcinoma: a randomized clinical trial. Journal of cancer research and clinical oncology 2006, 132(7):458-465.

8. Yang Y, Zhou Y, Hou J, Bai C, Li Z, Fan J, Ng IOL, Zhou W, Sun H, Dong Q et al: Hepatic IFIT3 predicts interferon-a therapeutic response in patients of hepatocellular carcinoma. Hepatology 2017, 66(1):152-166.

9. Hou J, Zhou Y, Zheng Y, Fan J, Zhou W, Ng IO, Sun H, Qin L, Qiu S, Lee JM et al: Hepatic RIG-I predicts survival and interferon-a therapeutic response in hepatocellular carcinoma. Cancer Cell 2014, 25(1):49-63.

10. Zhu WP, Liu ZY, Zhao YM, He XG, Pan Q, Zhang N, Zhou JM, Wang LR, Wang M, Zhan DH: Dihydropyrimidine dehydrogenase predicts survival and response to interferon- $\mathrm{a}$ in hepatocellular carcinoma. Cell Death \& Disease 2018, 9(2):69.

11. Salmena L, Poliseno L, Tay Y, Kats L, Pandolfi PP: A ceRNA hypothesis: the Rosetta Stone of a hidden RNA language? Cell 2011, 146(3):353-358.

12. Kimura T, Jiang S, Yoshida N, Sakamoto R, Nishizawa M: Interferon-alpha competing endogenous RNA network antagonizes microRNA-1270. Cellular and molecular life sciences : CMLS 2015, 72(14):2749-2761.

13. Carnero E, Barriocanal M, Segura V, Guruceaga E, Prior C, Börner K, Grimm D, Fortes P: Type I Interferon Regulates the Expression of Long Non-Coding RNAs. Frontiers in immunology 2014, 5:548.

14. Bai Y, Long J, Liu Z, Lin J, Huang H, Wang D, Yang X, Miao F, Mao Y, Sang X et al: Comprehensive analysis of a ceRNA network reveals potential prognostic cytoplasmic IncRNAs involved in HCC progression. Journal of cellular physiology 2019, 234(10):18837-18848. 
15. Long J, Bai Y, Yang X, Lin J, Yang X, Wang D, He L, Zheng Y, Zhao H: Construction and comprehensive analysis of a ceRNA network to reveal potential prognostic biomarkers for hepatocellular carcinoma. Cancer cell international 2019, 19:90.

16. Cinova J, Palová-Jelínková L, Smythies LE, Cerná M, Pecharová B, Dvorák M, Fruhauf P, TlaskalováHogenová H, Smith PD, Tucková L: Gliadin peptides activate blood monocytes from patients with celiac disease. Journal of clinical immunology 2007, 27(2):201-209.

17. Langmead B, Trapnell C, Pop M, Salzberg SL: Ultrafast and memory-efficient alignment of short DNA sequences to the human genome. Genome biology 2009, 10(3):R25.

18. Kim D, Pertea G, Trapnell C, Pimentel H, Kelley R, Salzberg SL: TopHat2: accurate alignment of transcriptomes in the presence of insertions, deletions and gene fusions. Genome biology 2013, 14(4):R36.

19. Traugott $U$, Lebon P: Interferon-gamma and la antigen are present on astrocytes in active chronic multiple sclerosis lesions. Journal of the neurological sciences 1988, 84(2-3):257-264.

20. Fabry Z, Raine CS, Hart MN: Nervous tissue as an immune compartment: the dialect of the immune response in the CNS. Immunology today 1994, 15(5):218-224.

21. Corbin JG, Kelly D, Rath EM, Baerwald KD, Suzuki K, Popko B: Targeted CNS expression of interferongamma in transgenic mice leads to hypomyelination, reactive gliosis, and abnormal cerebellar development. Molecular and cellular neurosciences 1996, 7(5):354-370.

22. LaFerla FM, Sugarman MC, Lane TE, Leissring MA: Regional hypomyelination and dysplasia in transgenic mice with astrocyte-directed expression of interferon-gamma. Journal of molecular neuroscience : MN2000, 15(1):45-59.

23. McHugh J: Systemic lupus erythematosus: IFN drives synapse loss via microglia. Nature reviews Rheumatology 2017, 13(8):449.

24. Kim IJ, Beck HN, Lein PJ, Higgins D: Interferon gamma induces retrograde dendritic retraction and inhibits synapse formation. The Journal of neuroscience : the official journal of the Society for Neuroscience 2002, 22(11):4530-4539.

25. Reick C, Ellrichmann G, Thöne J, Scannevin RH, Saft C, Linker RA, Gold R: Neuroprotective dimethyl fumarate synergizes with immunomodulatory interferon beta to provide enhanced axon protection in autoimmune neuroinflammation. Experimental neurology 2014, 257:50-56.

26. Weidner JM, Jiang D, Pan XB, Chang J, Block TM, Guo JT: Interferon-induced cell membrane proteins, IFITM3 and tetherin, inhibit vesicular stomatitis virus infection via distinct mechanisms. Journal of virology 2010, 84(24):12646-12657.

27. Le Page C, Génin P, Baines MG, Hiscott J: Interferon activation and innate immunity. Reviews in immunogenetics 2000, 2(3):374-386.

28. Cha L, Berry CM, Nolan D, Castley A, Fernandez S, French MA: Interferon-alpha, immune activation and immune dysfunction in treated HIV infection. Clinical \& translational immunology 2014, 3(2):e10. 
29. Hu X, Ivashkiv LB: Cross-regulation of signaling pathways by interferon-gamma: implications for immune responses and autoimmune diseases. Immunity 2009, 31(4):539-550.

30. Guan Q, Ezzati P, Spicer V, Krokhin O, Wall D, Wilkins JA: Interferon y induced compositional changes in human bone marrow derived mesenchymal stem/stromal cells. Clinical proteomics 2017, 14:26.

31. Nalbant D, Youn H, Nalbant SI, Sharma S, Cobos E, Beale EG, Du Y, Williams SC: FAM20: an evolutionarily conserved family of secreted proteins expressed in hematopoietic cells. BMC genomics 2005, 6:11.

32. Chelius D, Baldwin MA, Lu X, Spencer EM: Expression, purification and characterization of the structure and disulfide linkages of insulin-like growth factor binding protein-4. The Journal of endocrinology 2001, 168(2):283-296.

33. Contois LW, Nugent DP, Caron JM, Cretu A, Tweedie E, Akalu A, Liebes L, Friesel R, Rosen C, Vary C et al: Insulin-like growth factor binding protein-4 differentially inhibits growth factor-induced angiogenesis. The Journal of biological chemistry 2012, 287(3):1779-1789.

34. Feliers D, Woodruff K, Abboud S: Potential role of insulin-like growth factor binding protein-4 in the uncoupling of bone turnover in multiple myeloma. British journal of haematology 1999, 104(4):715722.

35. Lee YY, Mok MT, Kang W, Yang W, Tang W, Wu F, Xu L, Yan M, Yu Z, Lee SD et al: Loss of tumor suppressor IGFBP4 drives epigenetic reprogramming in hepatic carcinogenesis. Nucleic Acids Res 2018, 46(17):8832-8847.

36. Xiao Y, Zhu S, Yin W, Liu X, Hu Y: IGFBP-4 expression is adversely associated with lung cancer prognosis. Oncology letters 2017, 14(6):6876-6880.

37. Bauer J, Bakke O, Morth JP: Overview of the membrane-associated RING-CH (MARCH) E3 ligase family. New biotechnology 2017, 38(Pt A):7-15.

38. Xie L, Dai H, Li M, Yang W, Yu G, Wang X, Wang P, Liu W, Hu X, Zhao M: MARCH1 encourages tumour progression of hepatocellular carcinoma via regulation of PI3K-AKT- $\beta$-catenin pathways. Journal of cellular and molecular medicine 2019, 23(5):3386-3401.

39. Fan J, Tian L, Li M, Huang SH, Zhang J, Zhao B: MARCH8 is associated with poor prognosis in nonsmall cell lung cancers patients. Oncotarget 2017, 8(64):108238-108248.

\section{Tables}

Table 1. The clinicopathological characteristics in 4 HCC patients. 


\begin{tabular}{lll}
\hline Parameter & Classification & Results \\
\hline Age (y) & Mean \pm SD (range) & $52 \pm 15(32-67)$ \\
Sex & $\mathrm{M} / \mathrm{F}$ & $4 / 0$ \\
Aetiology & $\mathrm{HBV}+$ & $4 / 0$ \\
Advanced fibrosis or cirrhosis & Y/N & $3 / 1$ \\
Child-Pugh scores & $\mathrm{A} / \mathrm{B} / \mathrm{C}$ & $4 / 0 / 0$ \\
AJCC stage & $\mathrm{T} 1 / \mathrm{T} 2 / \mathrm{T} 3 / \mathrm{T} 4$ & $4 / 0 / 0 / 0$ \\
Tumor morphology & Nodular/Blocky pattern & $4 / 0$ \\
Capsule/pseudocapsule & Yes/No & $1 / 3$ \\
Tumor size (cm) & Mean \pm SD (range) & $3.0 \pm 1.1(2.0-4.5)$ \\
Grade & WD/MD/PD & $1 / 3 / 0$ \\
Microvascular invasion & Yes/No & $0 / 4$ \\
\hline
\end{tabular}

Abbreviations: HCC, hepatic carcinoma; AJCC, American Joint Committe on cancer; WD, well differentiated; MD, moderately-differentiated; PD, poorly-differentiated.

Table 2. Correlation analysis between MARCH3 and related genes and markers of immune cells in TIMER. 


\begin{tabular}{|c|c|c|c|c|c|}
\hline \multirow[b]{2}{*}{ Description } & \multirow[b]{2}{*}{ Gene markers } & \multicolumn{3}{|c|}{ MARCH3 } & \\
\hline & & None & & \multicolumn{2}{|l|}{ Purity } \\
\hline \multirow{3}{*}{ CD8+ T cell } & & Correlation & $\mathrm{P}$ & Correlation & $\mathrm{P}$ \\
\hline & CD8A & 0.318 & $* * *$ & 0.260 & $* * *$ \\
\hline & CD8B & 0.256 & $* * *$ & 0.190 & $* * *$ \\
\hline \multirow[t]{3}{*}{$\mathrm{T}$ cell } & CD3D & 0.409 & $* * *$ & 0360 & $* * *$ \\
\hline & CD3E & 0.432 & $* * *$ & 0.388 & $* * *$ \\
\hline & CD2 & 0.434 & $* * *$ & 0.388 & $* * *$ \\
\hline \multirow[t]{2}{*}{ B cell } & CD19 & 0.334 & $* * *$ & 0.283 & $* * *$ \\
\hline & CD79A & 0.390 & $* * *$ & 0.317 & $* * *$ \\
\hline \multirow[t]{2}{*}{ Monocyte } & CD86 & 0.455 & $* * *$ & 0.419 & $* * *$ \\
\hline & CSF1R & 0.367 & $* * *$ & 0.314 & $* * *$ \\
\hline \multirow[t]{3}{*}{ TAM } & CCL2 & 0.379 & $* * *$ & 0.319 & $* * *$ \\
\hline & CD68 & 0.329 & $* * *$ & 0.262 & $* * *$ \\
\hline & IL10 & 0.362 & $* * *$ & 0.309 & $* * *$ \\
\hline \multirow[t]{3}{*}{ M1 Macrophage } & NOS2 & 0.118 & $* * *$ & 0.107 & $* * *$ \\
\hline & IRF5 & 0.346 & $* * *$ & 0.344 & $* * *$ \\
\hline & PTGS2 & 0.465 & $* * *$ & 0.426 & $* * *$ \\
\hline \multirow[t]{3}{*}{ M2 Macrophage } & CD163 & 0.215 & $* * *$ & 0.148 & $* * *$ \\
\hline & VSIG4 & 0.268 & $* * *$ & 0.212 & $* * *$ \\
\hline & MS4A4A & 0.273 & $* * *$ & 0.208 & $* * *$ \\
\hline \multirow[t]{3}{*}{ Neutrophils } & CEACAM8 & 0.122 & $* * *$ & 0.116 & $* * *$ \\
\hline & ITGAM & 0.342 & $* * *$ & 0.303 & $* * *$ \\
\hline & CCR7 & 0.410 & $* * *$ & 0.357 & $* * *$ \\
\hline \multirow[t]{7}{*}{ Natural killer cell } & KIR2DL1 & -0.028 & $* * *$ & -0.054 & $* * *$ \\
\hline & KIR2DL3 & 0.110 & $* * *$ & 0.072 & $* * *$ \\
\hline & KIR2DL4 & 0.114 & $* * *$ & 0.082 & $* * *$ \\
\hline & KIR3DL1 & -0.022 & $* * *$ & -0.055 & $* * *$ \\
\hline & KIR3DL2 & 0.107 & $* * *$ & 0.086 & $* * *$ \\
\hline & KIR3DL3 & 0.049 & $* * *$ & 0.034 & $* * *$ \\
\hline & KIR2DS4 & 0.006 & $* * *$ & 0.010 & $* * *$ \\
\hline \multirow[t]{7}{*}{ Dendritic cell } & HLA-DPB1 & 0.379 & $* * *$ & 0.322 & $* * *$ \\
\hline & HLA-DQB1 & 0.284 & $* * *$ & 0.213 & $* * *$ \\
\hline & HLA-DRA & 0.333 & $* * *$ & 0.272 & $* * *$ \\
\hline & HLA-DPA1 & 0.347 & $* * *$ & 0.294 & $* * *$ \\
\hline & BDCA-1(CD1C) & 0.441 & $* * *$ & 0.390 & $* * *$ \\
\hline & BDCA-4(NRP1) & 0.314 & $* * *$ & 0.293 & $* * *$ \\
\hline & CD11c (ITGAX) & 0.458 & $* * *$ & 0.417 & $* * *$ \\
\hline \multirow[t]{5}{*}{ Th1 } & T-bet (TBX21) & 0.222 & $* * *$ & 0.141 & $* * *$ \\
\hline & STAT4 & 0.466 & $* * *$ & 0.430 & $* * *$ \\
\hline & STAT1 & 0.421 & $* * *$ & 0.391 & $* * *$ \\
\hline & IFN- $\gamma$ (IFNG) & 0.203 & $* * *$ & 0.160 & $* * *$ \\
\hline & TNF- $\alpha(T N F)$ & 0.403 & $* * *$ & 0.353 & $* * *$ \\
\hline \multirow[t]{4}{*}{ Th2 } & GATA3 & 0.444 & $* * *$ & 0.411 & $* * *$ \\
\hline & STAT6 & 0.135 & $* * *$ & 0.133 & $* * *$ \\
\hline & STAT5A & 0.339 & $* * *$ & 0.301 & $* * *$ \\
\hline & IL13 & 0.114 & $* * *$ & 0.094 & $* * *$ \\
\hline \multirow[t]{2}{*}{ Tfh } & BCL6 & 0.082 & $* * *$ & 0.096 & $* * *$ \\
\hline & IL21 & 0.103 & $* * *$ & 0.065 & $* * *$ \\
\hline \multirow[t]{2}{*}{ Th17 } & STAT3 & 0.290 & $* * *$ & 0.274 & $* * *$ \\
\hline & IL17A & 0.095 & $* * *$ & 0.101 & $* * *$ \\
\hline Treg & FOXP3 & 0.189 & $* * *$ & 0.166 & $* * *$ \\
\hline & CCR8 & 0.484 & $* * *$ & 0.448 & $* * *$ \\
\hline & STAT5B & 0.149 & $* * *$ & 0.198 & $* * *$ \\
\hline & TGFß (TGFB1) & 0.579 & $* * *$ & 0.543 & $* * *$ \\
\hline T cell exhaustion & PD-1 (PDCD1) & 0.437 & $* * *$ & 0.338 & $* * *$ \\
\hline & CTLA4 & 0.398 & $* * *$ & 0.356 & $* * *$ \\
\hline
\end{tabular}

Page 16/23 


\begin{tabular}{lllll} 
LAG3 & 0.235 & $* * *$ & 0.189 & $* * *$ \\
\hline TIM-3 (HAVCR2) & 0.481 & $* * *$ & 0.450 & $* * *$ \\
\hline GZMB & 0.083 & $* * *$ & 0.020 & $* * *$ \\
\hline
\end{tabular}

Abbreviations: $* \mathrm{P}<0.01 ; * * \mathrm{P}<0.001 ; * * * \mathrm{P}<0.0001$. The result of $\mathrm{P} \square 0.05$ means statistical significance.

Table 3. Correlation analysis between MARCH3 and relate genes and markers of $\mathrm{T}$ cell exhaustion in GEPIA.

\begin{tabular}{|c|c|c|c|c|c|}
\hline Description & $\begin{array}{l}\text { Gene markers } \\
\text { Tumor }\end{array}$ & & & $\mathrm{HCC}$ & Normal \\
\hline T cell exhaustion & PD-1 (PDCD1) & $\begin{array}{l}\mathbf{R} \\
0.43\end{array}$ & $\mathbf{P}$ & $\begin{array}{l}* * * \\
* * *\end{array}$ & $\begin{array}{lc}\mathbf{R} & \mathbf{P} \\
0.33 & 0.02\end{array}$ \\
\hline
\end{tabular}

Abbreviations: HCC, Hepatocellular carcinoma; Tumor, correlation analysis in tumor tissue of TCGA. Normal, correlation analysis in normal tissue of TCGA. *P $<0.01$; **P $<0.001$; *** $<0.0001$. The result of $\mathrm{P} \square 0.05$ means statistical significance.

\section{Figures}


a

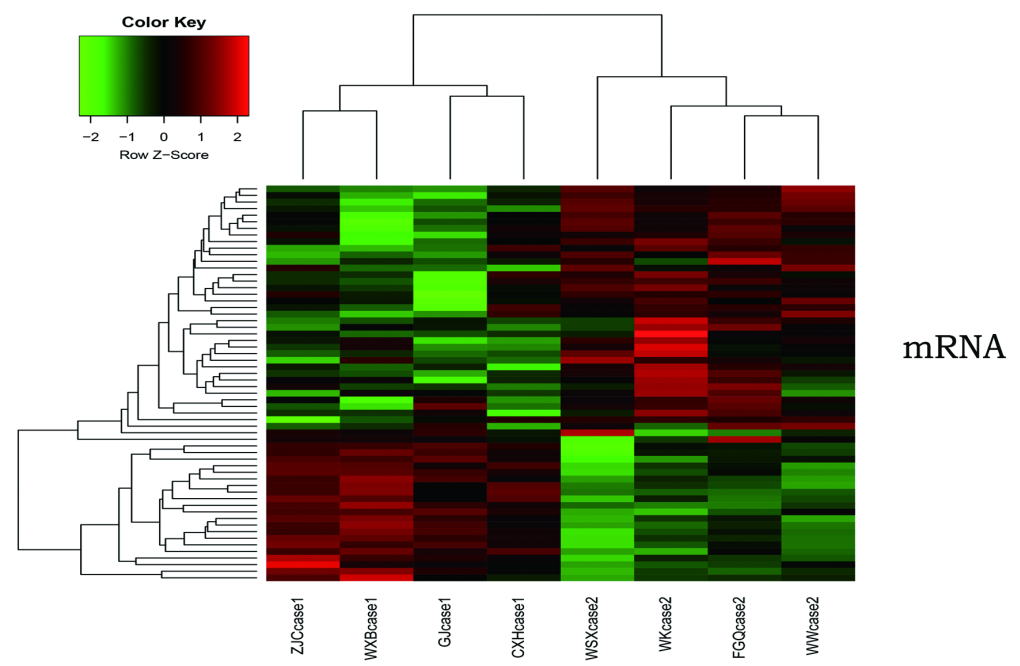

b

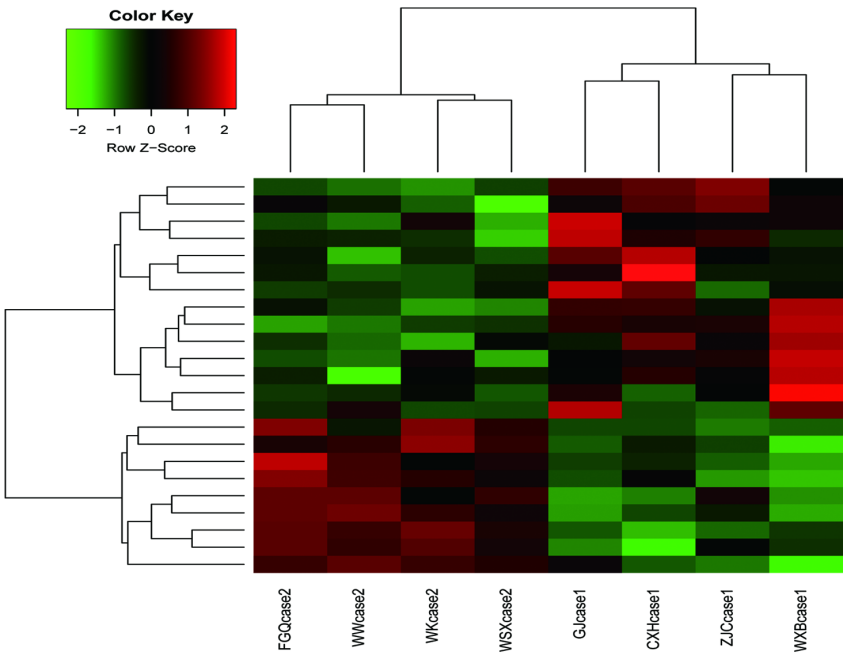

circRNA

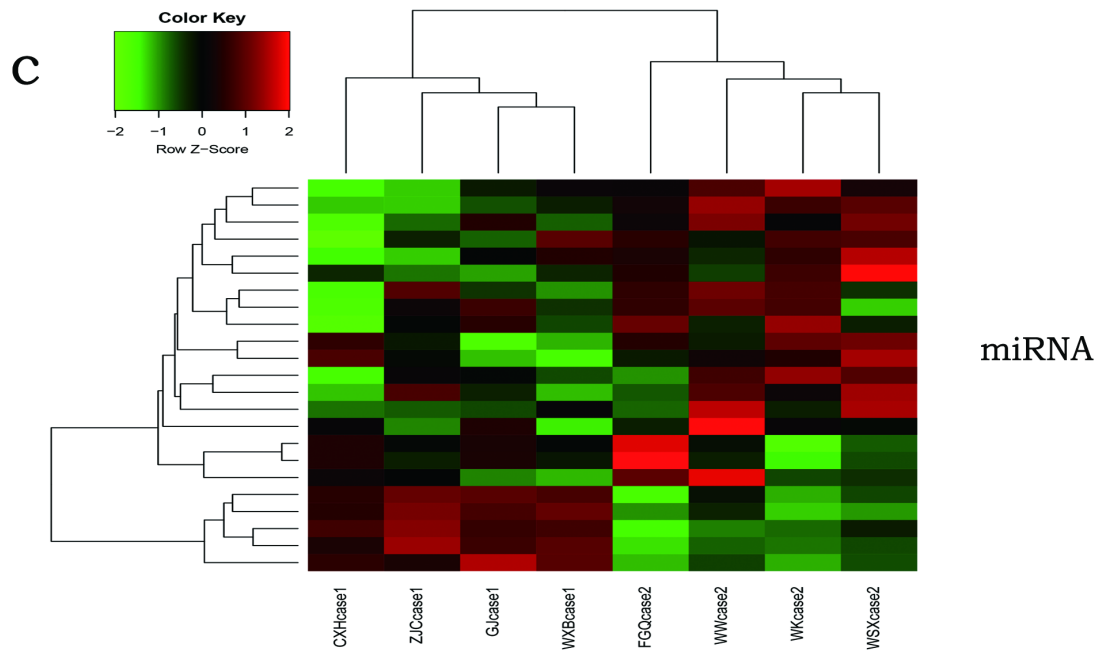

Figure 1

Significance and reliability of differentially expressed RNAs between the two groups. a mRNAs; $b$ circRNAs; c miRNAs. 
a
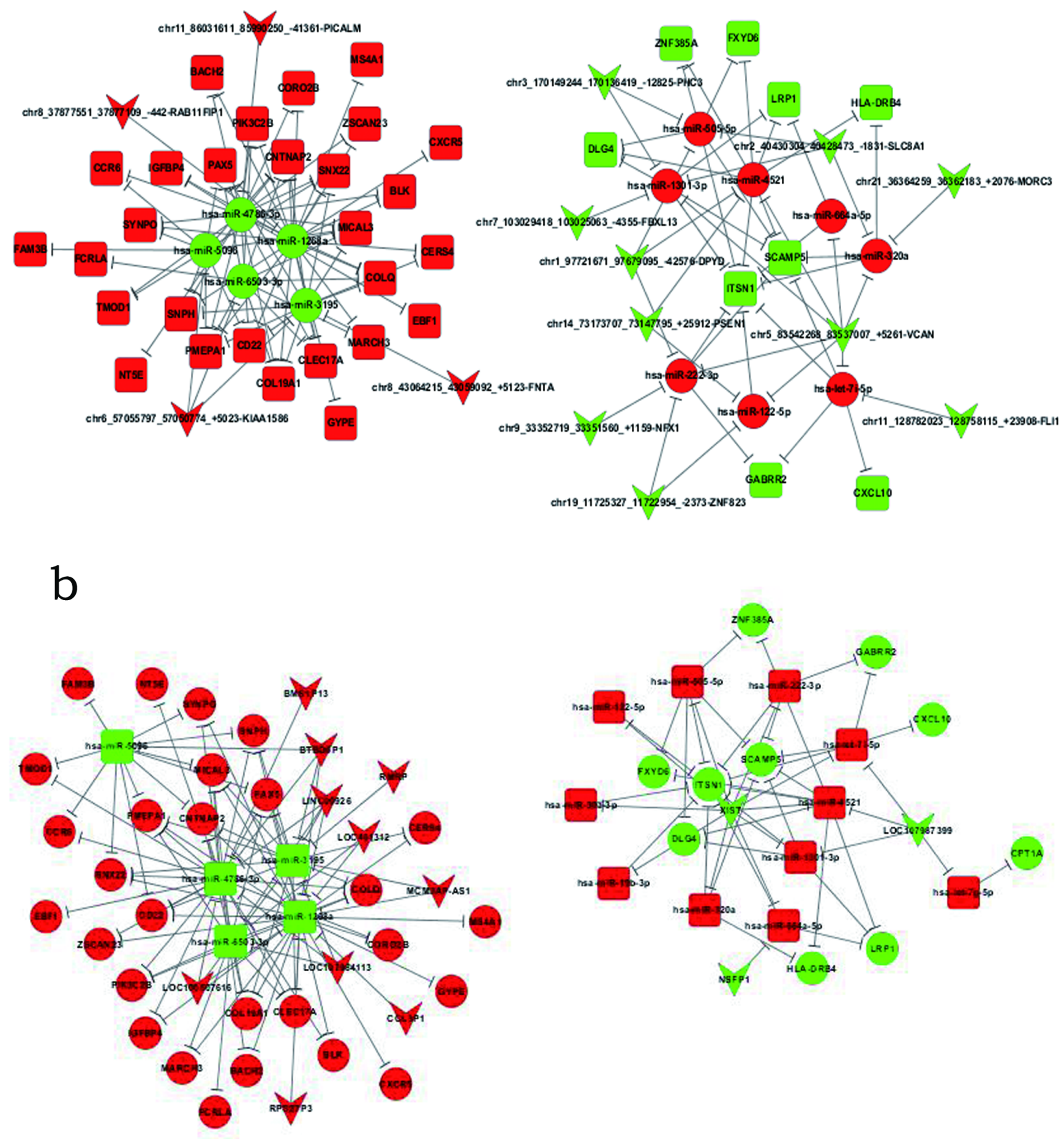

Figure 2

The ceRNA regulatory network under the IFN- $a$ therapy. a The circRNA-miRNA-mRNA network. $b$ The IncRNA-miRNA-mRNA network. 


\section{a}

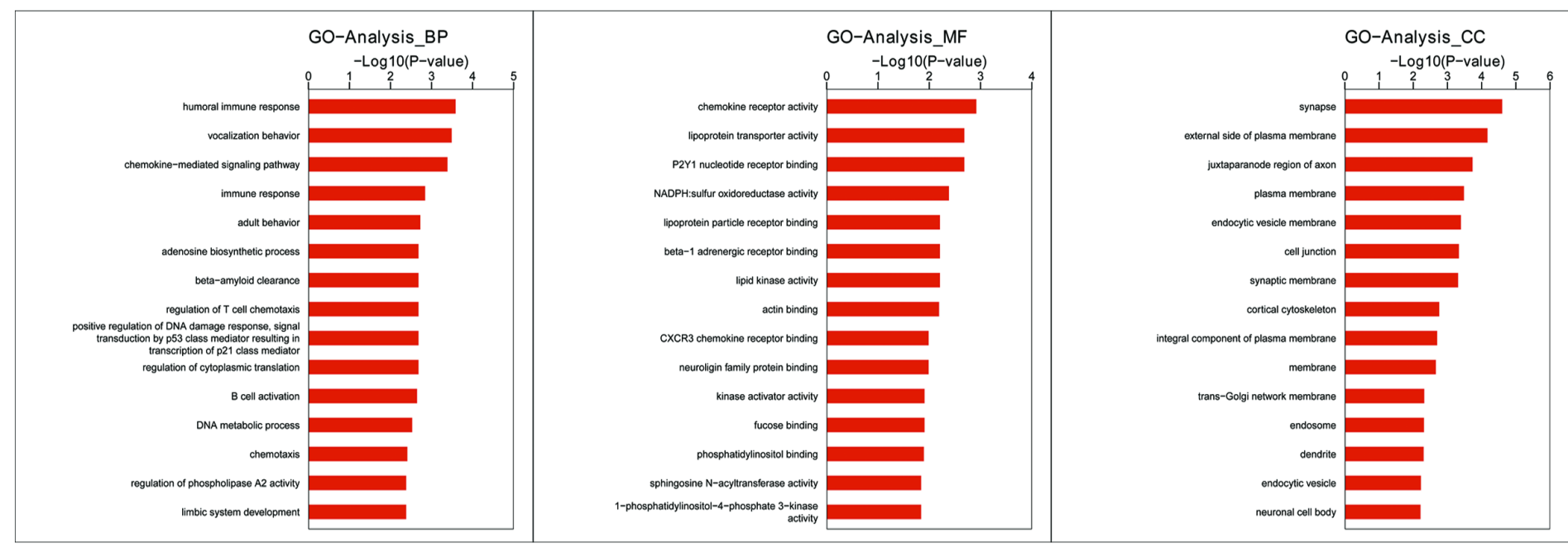

b

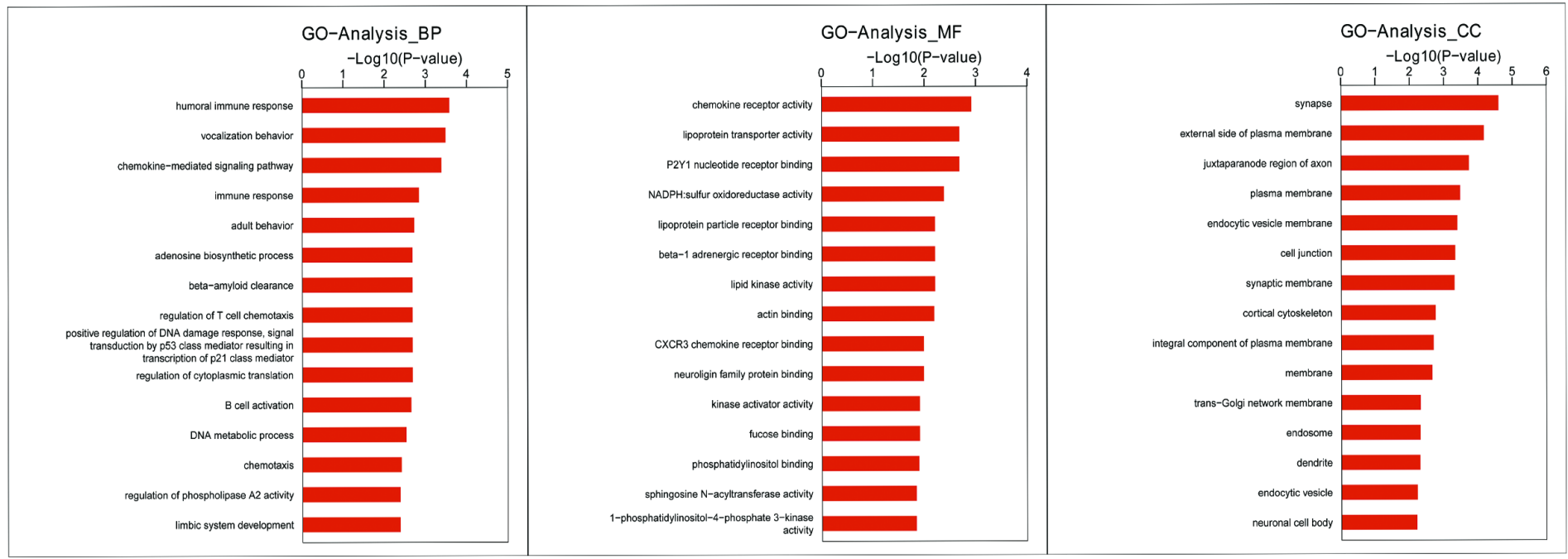

\section{Figure 3}

Gene oncology analysis for differentially expressed RNAs involved in the ceRNA network. a Genes in the circRNA-miRNA-mRNA network. b Genes in the IncRNA-miRNA-mRNA network. 
a

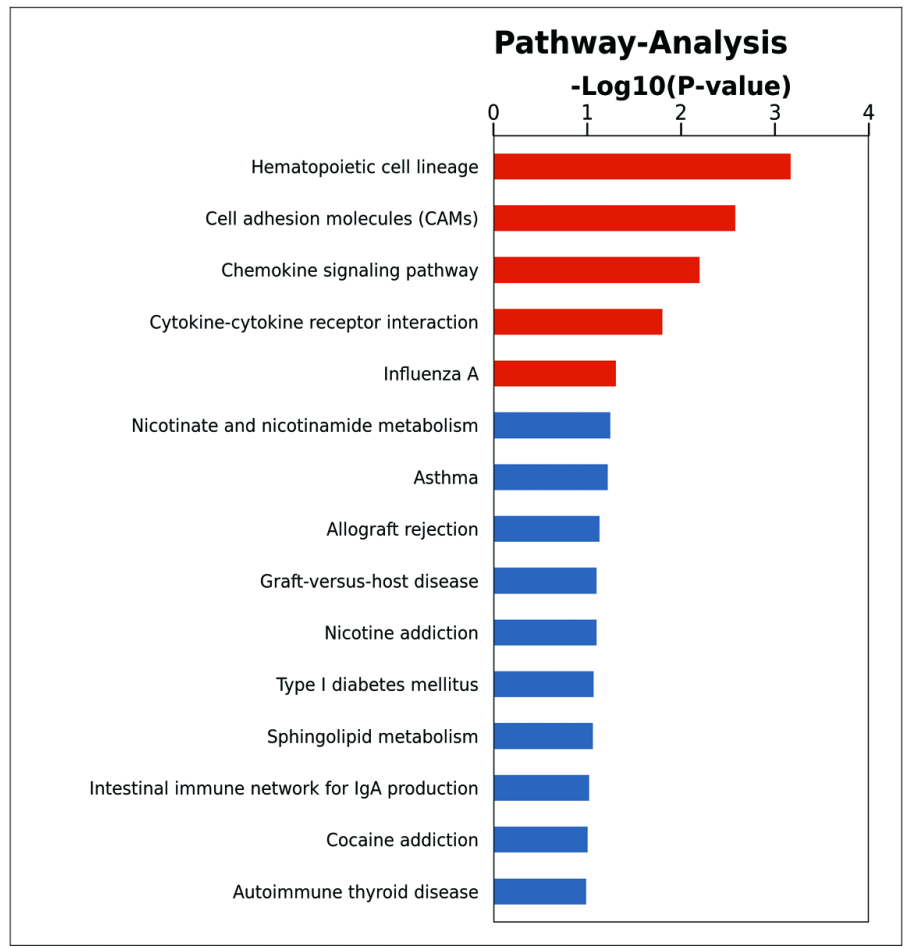

b

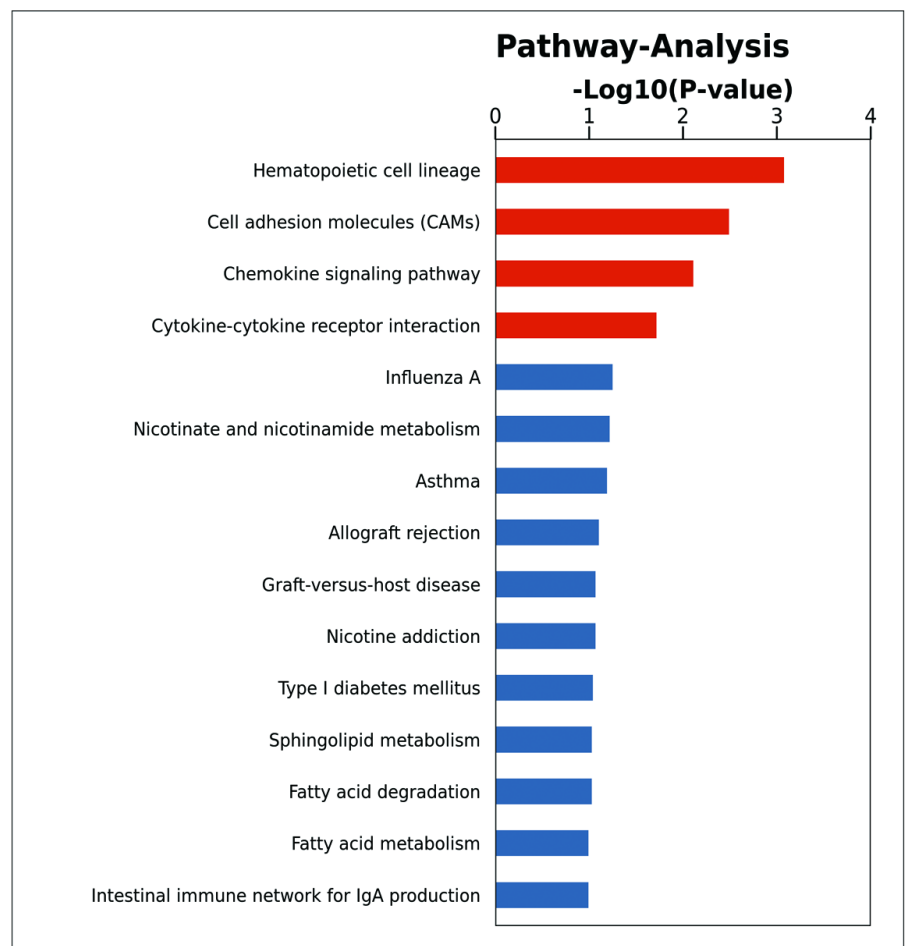

\section{Figure 4}

The enriched KEGG pathway of differentially expressed RNAs involved in the ceRNA network under the IFN-a therapy. a The up-regulated RNAs networks $\llbracket b$ The down-regulated RNAs networks 
a

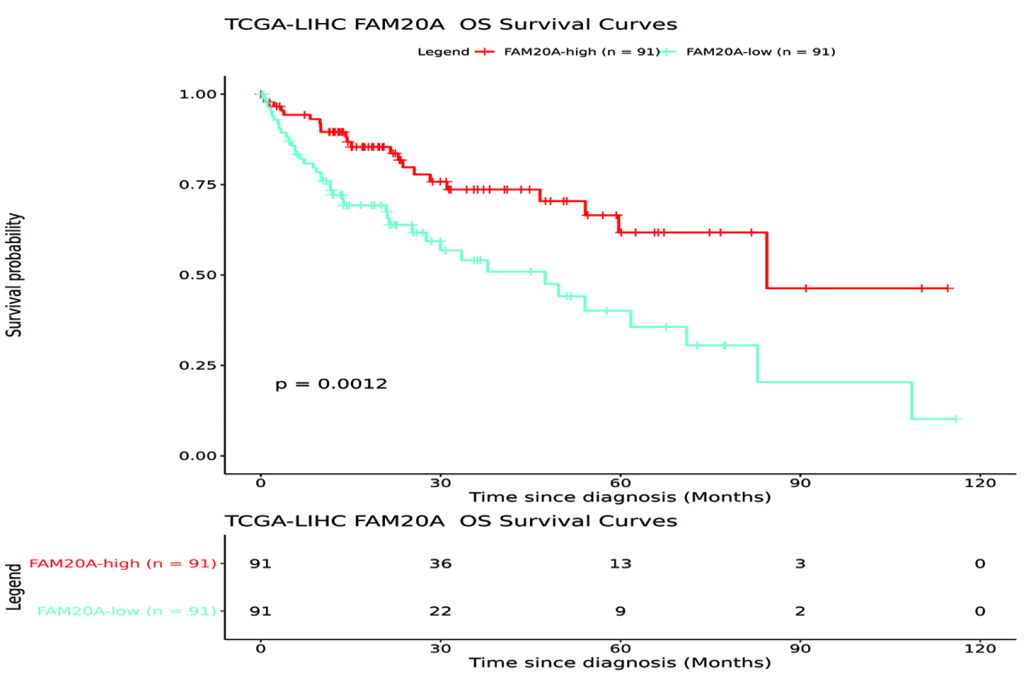

b

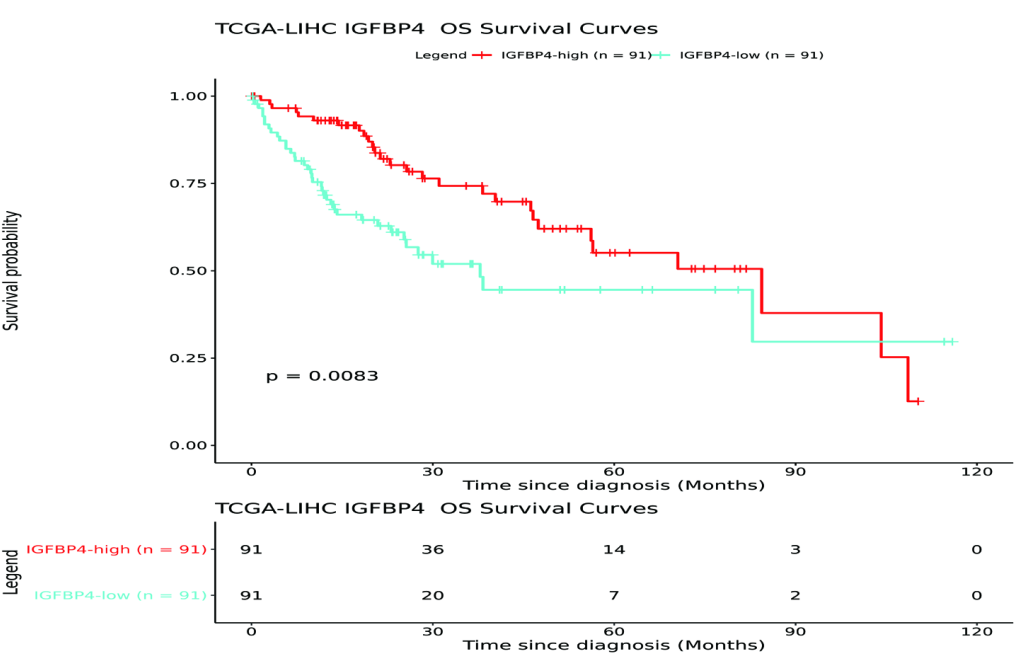

C

TCGA-LIHC MARCH3 OS Survival Curves

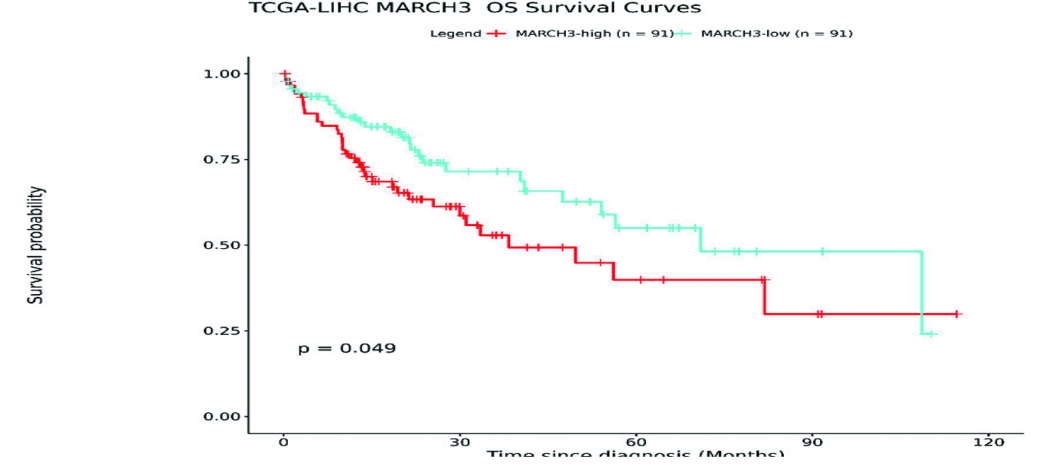

Figure 5

Kaplan-Meier survival curves for differentially expressed genes most relevant to overall survival of HCC patients. a FAM2OA; b IGFBP4; c MARCH3. 

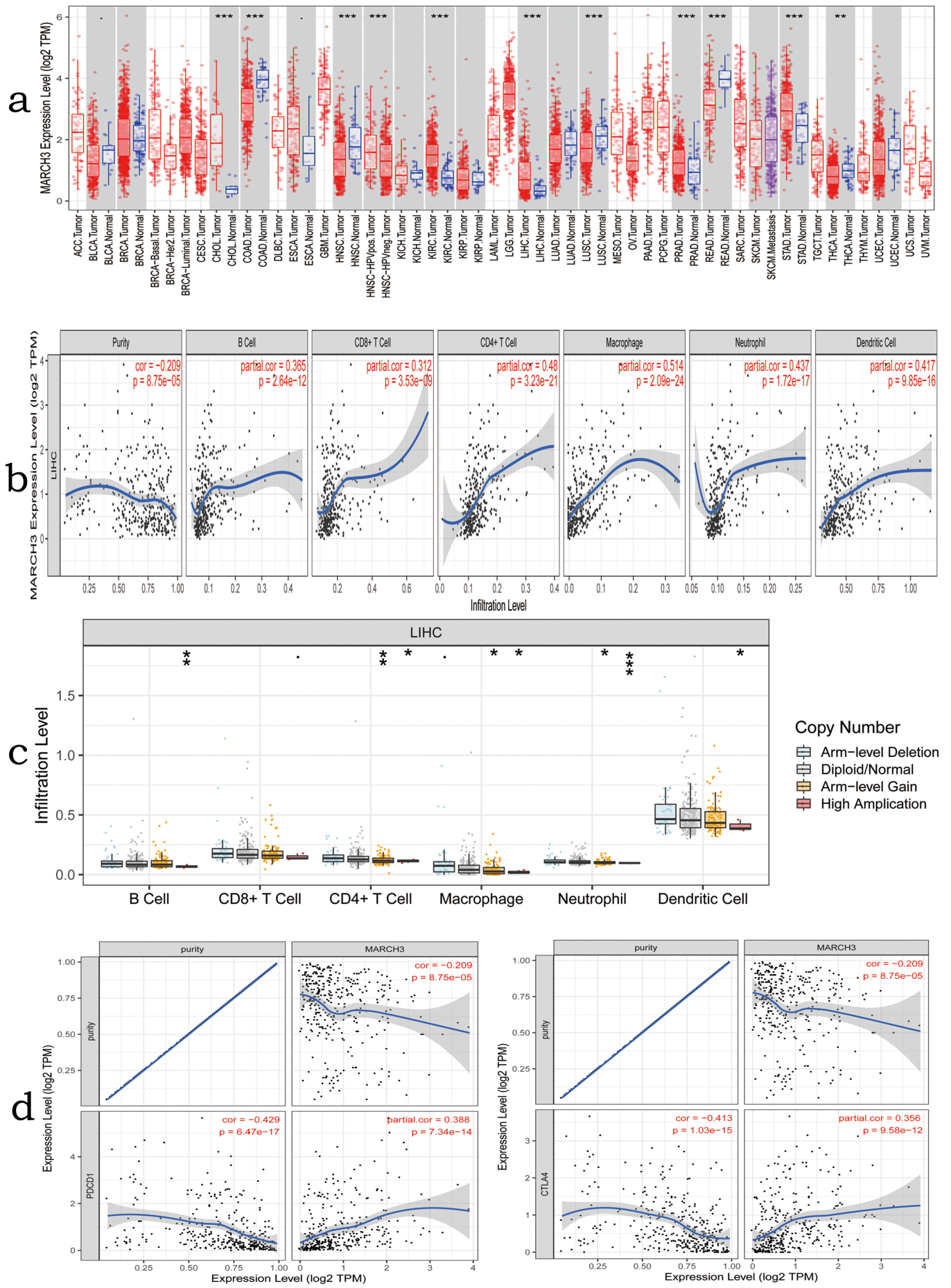

Figure 6

Correlations between MARCH3 expression and immune infiltration in HCC. a Expression of MARCH3 in various cancers; $b$ Correlations of MARCH3 expression with immune infiltration level in HCC; c Correlations of MARCH3 CNV with immune infiltration level in HCC; $d$ Correlations between marker sets of T cell exhaustion in HCC. 